\section{OPEN ACCESS}

Edited by:

Dinesh Kumar Bhugra, King's College London, United Kingdom

Reviewed by:

Simon Ching Lam, Hong Kong Polytechnic University,

Hong Kong

Reina Granados,

University of Granada, Spain

*Correspondence:

Yongsheng Jiang

ysjiang.china@163.com

Furong Wang

wangfurong.china@163.com

Specialty section: This article was submitted to Mood and Anxiety Disorders,

a section of the journal

Frontiers in Psychiatry

Received: 24 June 2020 Accepted: 12 October 2020 Published: 05 November 2020

Citation:

Cao H, Zuo C, Li G, Huang Y, Li L,

Huang S, Zhao J, Liu J, Jiang Y and Wang F (2020) A Cross-Sectional Study of Psychological Status in Different Epidemic Areas in China After the COVID-19 Outbreak. Front. Psychiatry 11:575705. doi: 10.3389/fpsyt.2020.575705

\title{
A Cross-Sectional Study of Psychological Status in Different Epidemic Areas in China After the COVID-19 Outbreak
}

\begin{abstract}
Huan Cao ${ }^{1}$, Chengchao Zuo ${ }^{1}$, Guo Li $^{1}$, Yaqi Huang ${ }^{1}$, Ling Li ${ }^{1}$, Shu Huang ${ }^{1}$, Jianling Zhao ${ }^{1}$, Jingjing Liu ${ }^{1}$, Yongsheng Jiang ${ }^{2 \star}$ and Furong Wang ${ }^{1 *}$
\end{abstract}

${ }^{1}$ Department of Neurology, Tongji Hospital, Tongji Medical College, Huazhong University of Science and Technology, Wuhan, China, ${ }^{2}$ Cancer Center of Tongji Hospital, Tongji Medical College, Huazhong University of Science and Technology, Wuhan, China

Background: The outbreak of coronavirus disease 2019 in Wuhan, Hubei Province, China has seriously affected people's mental health. We aimed to assess the psychological impact of the coronavirus disease 2019 on health care workers and non-health care workers in three different epidemic areas in China and to identify independent risk factors.

Methods: We surveyed 1,020 non-health care workers and 480 health care workers in Wuhan, other cities in Hubei except Wuhan and other provinces in China except Hubei.

Results: Health care workers in Hubei had higher levels of anxiety and depression than non-health care workers ( $p<0.05)$, but there was no such difference in other provinces in China except Hubei $(p>0.05)$. Compared with other regions, health care workers in Wuhan was more anxious ( $p<0.05$ ), and this anxiety may be caused by concerns about occupational exposure and wearing protective clothing for a long time daily; health care workers in Hubei had more obvious depression $(p<0.05)$, which may be associated with long days participating in epidemic work and wearing protective clothing for a long time daily. Meanwhile, $62.5 \%$ of health care workers were proud of their work. The anxiety and depression of non-health care workers in Wuhan were also the most serious.

Conclusions: In Wuhan, where the epidemic is most severe, levels of anxiety and depression seem to be higher, especially among health care workers. This information may help to better prepare for future events.

\section{Keywords: COVID-19, depression, anxiety, psychological stress, health care workers}

\section{INTRODUCTION}

In December 2019, a new type of coronavirus disease 2019 (COVID-19) appeared in Wuhan and spread rapidly domestically and internationally, posing a global health emergency. As of March 7, 2020, more than 80,000 people in China have been diagnosed with COVID-19, and more than 100,000 people worldwide have been diagnosed. Confirmed cases have been reported in all provinces of China. The growing number of confirmed and suspected cases, as well as the geographical spread of the disease, has raised public concern about infection, triggering public panic and psychological stress $(1,2)$. 
Studies have shown that the public exhibited significant mental disorders such as fear, stress, sleep disorders, anxiety, and depression during the COVID-19 epidemic (3). Based on experiences of severe acute respiratory syndrome (SARS), it was suggested that these psychological problems were closely related to the severity of the epidemic (4). The stress effects of infectious diseases may be qualitatively distinct from those of other disasters, especially for health care workers (HCWs) (5). HCWs, overworked at the front line, have a high risk of occupational exposure and infection (6). Infection rates among HCWs in various countries during SARS ranged from 3 to $51 \%$ (7). As of February 17, 2020, Chinese officials reported that more than 3,000 HCWs had been infected with COVID-19. Another important reason for the psychological burden on HCWs is the greater fear of their family members being at a higher risk of infection (8-11). Therefore, when new epidemics break out, HCWs often suffer from huge psychological health problem, such as anxiety and depression $(12,13)$.

The psychological disorder is not only a temporary process and it may persist for a few years after the epidemic ends (14). Therefore, in addition to identify pathogenic factors, pathophysiology, clinical manifestations, diagnosis and treatment, it is also important to study the impact of COVID19 outbreak on people's mental health. Understanding the psychological impact during an outbreak, such as anxiety levels, can not only help predict key behavioral outcomes (e.g., wearing a face-mask) (4), but may also have important implications for future psychological and behavioral research and public health interventions related to respiratory communicable diseases. Although there has been some research in this area, we have also studied the psychological conditions of the general public and HCWs in three different epidemic areas in China during the outbreak period. Specifically, this study investigated the levels of anxiety and depression of HCWs and non-HCWs, determined the impact of the severity of the epidemic on people in corresponding regions (The epidemic is worst in Wuhan, followed by other cities in Hubei except Wuhan, and the situation in other provinces in China except Hubei are better than these two regions), and evaluated the work perceptions of HCWs.

\section{METHODS}

\section{Participants}

People aged 18 or above from all walks of life in Wuhan, other cities in Hubei except Wuhan, and other provinces in China except Hubei were welcome to join in the survey. Other cities in Hubei except Wuhan include 12 cities, autonomous prefecture in Hubei. Other provinces in China except Hubei include 30 provinces, autonomous regions and municipalities in the mainland of China. The classification was based on their geographic location, which was also closely related to the severity of the epidemic (e.g., Wuhan, other regions inside Hubei Province, and regions outside Hubei Province). The sample size was calculated to be 1,568. All participants are divided into HCWs (i.e., doctors and nurses) and non-HCWs (Others except doctors and nurses). Sociodemographic characteristics including name, gender, age, education level, occupation, annual family income, type of medical insurance, household registration location, current city.

\section{Data Collection}

The questionnaire was collected through social media WeChat (15) (Tencent, Shenzhen, China) using an online survey platform Sojump (Changsha ran Xing InfoTech Ltd.,) during February 6th to 13 th, 2020, when the epidemic was in the outbreak period, the situation was chaotic (16-18) and the number of people infected with COVID-19 was escalating. The convenience sampling was adopted. After the researchers explained the purpose of the study, 1,568 volunteers completed the questionnaire-based surveys, of which 1,500 were valid. Among the three regions, there were 1,020 questionnaires for non-HCWs and 480 questionnaires for HCWs. Each person can only answer one questionnaire. Sojump APP can set the number of answers, which can be set as each WeChat account is only allowed to answer once, and each IP is only allowed to answer once. Informed consent is exempt. Ethical approval was received from the Institutional Review Board (IRB) of the University of Huazhong university of science and technology. The ethical number is TJ-IRB20200322.

\section{Basic Information Survey}

A self-designed questionnaire was used to determine the relevant factors. Basic information about COVID-19 including the degree of attention to the COVID-19, whether relatives or friends have the COVID-19, and the current status (healthy home quarantine, suspected isolation, mild illness isolation, fever outpatient service, hospitalization, square cabin hospital). For HCWs, we added some additional items that can reflect their occupational exposure risk, work intensity, worries and current thoughts about their occupation, including operating post (fever outpatient service, inpatient department, square cabin hospital, others), the number of days participating in epidemic work, rest days in the past month, the average working hours per week, daily wear protective clothing time (hours), whether you are worried about occupational exposure (none, occasionally, often), whether you are worried about your family's cross-infection because of you, and the main feelings about your career (pride, fear, pessimism, it doesn't matter).

\section{Anxiety and Depression Status Assessment}

The assessment scale was composed of the Hamilton Anxiety Rating Scale (HAMA; Hamilton, 1959) and Hamilton Depression Rating Scale (HAMD; Hamilton, 1960), both of which had high reliability reported in the literature (the reliability of HAMA ranged between 0.82 and 0.94 ; the reliability of HAMD ranged between 0.81 and 0.98) (19-21). In this study, HAMA and HAMD also showed good internal consistency, with Cronbach's $\alpha$ coefficients of the total scale being 0.94 and 0.90 respectively. HAMA includes 14 items, each with $0 \sim 4$ points, corresponding to asymptomatic, mild, moderate, severe, and extremely severe. Total score $\geq 29$ points, there may be severe anxiety; Total score $21 \sim 28$ points, there must be significant anxiety; Total score $14 \sim 20$ points, there must be anxiety; Total score $7 \sim 13$ points, there may be anxiety; Total score $<7$ points, no anxiety symptoms. HAMD includes 24 items, a score of 0 in each item 
TABLE 1 | Sociodemographic characteristics.

\begin{tabular}{lccc}
\hline & HCWs & non-HCWs & $\boldsymbol{p}$ \\
\hline Sex, $n$ (\%) & & & $<0.05$ \\
Female & $394(82.08)$ & $628(61.57)$ & \\
Male & $86(17.92)$ & $392(38.43)$ & \\
Age & $35.29 \pm 8.85$ & $31.87 \pm 11.25$ & $<0.05$ \\
Education, $n$ (\%) & & & $<0.05$ \\
Bachelor degree or above & $398(82.92)$ & $446(43.73)$ & \\
Junior college or technical secondary & $82(17.08)$ & $524(51.37)$ & \\
school & & & \\
HAMA & $4.30 \pm 5.95$ & $3.29 \pm 5.92$ & $<0.05$ \\
HAMD & $8.19 \pm 7.97$ & $6.35 \pm 7.93$ & $<0.05$ \\
\hline
\end{tabular}

represents no symptoms. Total score > 35: major depression; Total score 21 35: definitely having depression; Total score $8 \sim 20$ : possible depression; Total score $<8$, normal.

\section{Statistical Analysis}

All statistical analyses were conducted using SPSS 19. The measurement data are expressed as mean \pm standard deviation, independent sample $t$-test was used for comparison between the two groups. The counting data were represented by the number of cases (\%), and the comparison was tested using $\chi^{2}$. Finally, the risk of anxiety and depression was further analyzed by multivariate logistic regression (using likelihood ratio estimation). Two-sided test with $p<0.05$ was considered statistically significant.

\section{RESULTS}

\section{Sociodemographic Characteristics}

Table 1 shows the main demographic characteristics of the participants and their scores on measures of anxiety and depression. A total of 1,568 questionnaires were collected, of which 1,500 were valid and the effective rate was $95.66 \%$. Among them, female accounted for $82.08 \%$ of HCWs and $61.57 \%$ of nonHCWs. The mean age of HCWs and non-HCWs were $35.29 \pm$ 8.85 years and $31.87 \pm 11.25$ years, respectively. The education levels of HCWs were Bachelor degree or above (82.92\%) and Junior college or technical secondary school (17.08\%). The education level of non-HCWs were Bachelor degree or above $(43.73 \%)$ and Junior college or technical secondary school (51.37\%), the other $4.9 \%$ including primary, middle and high school. The average HAMA scores for HCWs and non-HCWs were $4.30 \pm 5.95$ and $3.29 \pm 5.92$, and the average HAMD scores for HCWs and non-HCWs were 8.19 \pm 7.97 and $6.35 \pm 7.93$.

\section{Anxiety}

In general, HCWs were more anxious than non-HCWs $\left(\chi^{2}=\right.$ 9.36, $p<0.05$, Table 2). HAMA score less than 7 is considered to have no anxiety, accounting for $75.83 \%$ in HCWs and $82.55 \%$ in non-HCWs. Similarly, in Wuhan $\left(\chi^{2}=7.32, p<0.05\right)$ and other cities in Hubei except Wuhan $\left(\chi^{2}=6.40, p<0.05\right)$, HCWs was also more anxious than non-HCWs (Table 2). But in
TABLE 2 | Comparison of anxiety among HCWs and non-HCWs.

\begin{tabular}{|c|c|c|c|c|c|c|}
\hline & \multirow[t]{2}{*}{$N$} & \multicolumn{3}{|c|}{$\begin{array}{l}\text { Composition of HAMA } \\
\qquad n(\%)\end{array}$} & \multirow[b]{2}{*}{$x^{2}$} & \multirow[b]{2}{*}{$p$} \\
\hline & & $<7$ & $7 \sim 13$ & $>14$ & & \\
\hline All samples & 1,500 & 1206(80.4) & 199(13.27) & $95(6.33)$ & & \\
\hline HCWs & 480 & $364(75.83)$ & $78(16.25)$ & $38(7.92)$ & & \\
\hline non-HCWs & 1,020 & $842(82.55)$ & $121(11.86)$ & $57(5.59)$ & 9.36 & $<0.05$ \\
\hline \multicolumn{7}{|l|}{ Wuhan } \\
\hline HCWs & 134 & $83(61.94)$ & $32(23.88)$ & $19(14.18)$ & & \\
\hline non-HCWs & 208 & $157(75.48)$ & $34(16.35)$ & $17(8.17)$ & 7.32 & $<0.05$ \\
\hline \multicolumn{7}{|c|}{ Other cities in Hubei except Wuhan } \\
\hline HCWs & 207 & 161(77.78) & 33(15.94) & $13(6.28)$ & & \\
\hline non-HCWs & 361 & $310(85.87)$ & $34(9.42)$ & $17(4.71)$ & 6.40 & $<0.05$ \\
\hline \multicolumn{7}{|c|}{ Other provinces in China except Hubei } \\
\hline HCWs & 139 & 120(86.33) & 13(9.35) & $6(4.32)$ & & \\
\hline non-HCWs & 451 & $375(83.15)$ & 53(11.75) & $23(5.10)$ & 0.81 & $>0.05$ \\
\hline
\end{tabular}

other provinces in China except Hubei, there was no significant difference in anxiety levels between HCWs and non-HCWs $\left(\chi^{2}\right.$ $=0.81, p>0.05$, Table 2).

The anxiety levels of HCWs in other cities in Hubei except Wuhan $\left(\chi^{2}=10.95, p<0.05\right)$ and other provinces in China except Hubei $\left(\chi^{2}=21.44, p<0.05\right)$ were significantly lower than that in Wuhan, but there was no significant difference in HCWs anxiety levels between other cities in Hubei except Wuhan and other provinces in China except Hubei $\left(\chi^{2}=4.05\right.$, $p>0.05$, Table 3). Different from HCWs, although the anxiety level of non-HCWs in Wuhan was higher than that in other cities in Hubei except Wuhan $\left(\chi^{2}=9.69, p<0.05\right)$, it was not significantly higher than that in other provinces in China except Hubei $\left(\chi^{2}=5.53, p>0.05\right)$. Moreover, there was no significant difference between other cities in Hubei except Wuhan and other provinces in China except Hubei $\left(\chi^{2}=1.26, p>0.05\right.$, Table 3).

Next, we explored the reasons for the different anxiety levels of HCWs in different regions. We used the multivariable logistic regression analysis to test the variables of sex, age, whether you often worry about occupational exposure, whether you are worried about your family's cross-infection because of you, daily wearing protective clothing time (hours) and participation in epidemic work(days). It was found that whether you often worry about occupational exposure (OR 2.833; 95\% CI 1.274-6.298) and daily wearing protective clothing time (hours) (OR 1.086; 95\% CI 1.034-1.140) were the independent risk factors $(<0.05)$ (Table 4).

\section{Depression}

Compared with non-HCWs, HCWs showed significant depression $\left(\chi^{2}=36.03, p<0.05\right.$, Table 5$)$. Similar to the anxiety condition, the depression levels of HCWs were more serious than that of non-HCWs in Wuhan $\left(\chi^{2}=9.67, p<0.05\right)$ and other cities in Hubei except Wuhan $\left(\chi^{2}=30.52, p<0.05\right)$. There was no significant difference between other cities in Hubei 
TABLE 3 | Comparison of anxiety in different areas.

\begin{tabular}{|c|c|c|c|c|c|c|c|c|}
\hline & \multirow[t]{2}{*}{$N$} & \multicolumn{3}{|c|}{$\begin{array}{l}\text { Composition of HAMA } \\
\qquad n(\%)\end{array}$} & \multirow[b]{2}{*}{$x^{2}$} & \multirow[b]{2}{*}{$P^{*}$} & \multirow[b]{2}{*}{$x^{2}$} & \multirow[b]{2}{*}{$P \#$} \\
\hline & & $<7$ & $7 \sim 13$ & $>14$ & & & & \\
\hline \multicolumn{9}{|l|}{ HCWs } \\
\hline Wuhan & 134 & 83(61.94) & $32(23.88)$ & $19(14.18)$ & & & & \\
\hline Other cities in Hubei except Wuhan & 207 & $161(77.78)$ & 33(15.94) & 13(6.28) & 10.95 & $<0.05^{\star}$ & & \\
\hline $\begin{array}{l}\text { Other provinces in China except Hubei } \\
\text { non-HCWs }\end{array}$ & 139 & 120(86.33) & $13(9.35)$ & $6(4.32)$ & 21.44 & $<0.05^{\star}$ & 4.05 & $>0.05$ \\
\hline Wuhan & 208 & $157(75.48)$ & $34(16.35)$ & $17(8.17)$ & & & & \\
\hline Other cities in Hubei except Wuhan & 361 & $310(85.87)$ & $34(9.42)$ & $17(4.71)$ & 9.69 & $<0.05^{\star}$ & & \\
\hline Other provinces in China except Hubei & 451 & 375(83.15) & $53(11.75)$ & 23(5.10) & 5.53 & $>0.05$ & 1.26 & $>0.05$ \\
\hline
\end{tabular}

$P^{\star}$ compared with Wuhan, P\# comparison between other cities in Hubei except Wuhan and other provinces in China except Hubei.

TABLE 4 | Risk of anxiety: logistic regression analysis.

\begin{tabular}{lcc}
\hline Variable & OR (95\% Cl) & $\boldsymbol{p}$ \\
\hline Male & $1.254(0.455-3.460)$ & 0.662 \\
Age & $1.006(0.964-1.050)$ & 0.784 \\
Whether you often worry about occupational & $2.833(1.274-6.298)$ & 0.011 \\
exposure (yes) & & \\
Whether you are worried about your family's & $0.272(0.035-2.116)$ & 0.214 \\
cross-infection because of you (no) & & \\
Daily wear protective clothing time (hours) & $1.086(1.034-1.140)$ & 0.001 \\
Participation in epidemic work(days) & $1.012(0.985-1.039)$ & 0.397 \\
\hline
\end{tabular}

OR, odds ratio; $\mathrm{Cl}$, confidence interval.

except Wuhan and other provinces in China except Hubei $\left(\chi^{2}=\right.$ $0.56, p>0.05$, Table 5).

Further analysis showed that, among the three regions, HCWs in Wuhan $\left(\chi^{2}=25.82, p<0.05\right)$ and other cities in Hubei except Wuhan $\left(\chi^{2}=11.95, p<0.05\right)$ had significant differences in depression levels compared with those in other provinces in China except Hubei (Table 6). Unlike anxiety, non-HCWs in other cities in Hubei except Wuhan $\left(\chi^{2}=22.45, p<0.05\right)$ and other provinces in China except Hubei $\left(\chi^{2}=20.07, p<0.05\right)$ had lower depression levels than those in Wuhan. There was no difference between other cities in Hubei except Wuhan and other provinces in China except Hubei $\left(\chi^{2}=1.02, p>0.05\right.$, Table 6).

Finally, we explored the causes of different levels of depression in HCWs in different regions. It was found that daily wearing protective clothing time (hours) (OR 1.100; 95\% CI 1.040-1.163) and participation in epidemic work(days) (OR 1.030; 95\% CI 1.006-1.054) were the independent risk factors (Table 7).

\section{DISCUSSION}

At the end of 2019, the outbreak of COVID-19 swept China and affected the whole world. Due to the severe situation of the epidemic, it was listed as a public health emergency of international concern (PHEIC) by WHO on January 30, 2020 (22). In China, people's normal work and life were interrupted,
TABLE 5 | Comparison of depression among HCWs and non-HCWs.

\begin{tabular}{|c|c|c|c|c|c|c|c|}
\hline & \multirow[b]{2}{*}{$\boldsymbol{N}$} & \multicolumn{4}{|c|}{$\begin{array}{c}\text { Composition of HAMD } \\
\qquad n(\%)\end{array}$} & \multirow[t]{2}{*}{$x^{2}$} & \multirow[t]{2}{*}{$p$} \\
\hline & & $<8$ & $8 \sim 20$ & 21 35 & $>35$ & & \\
\hline All samples & 1500 & 1036(69.07) & $343(22.87)$ & 103(6.87) & 18(1.20) & & \\
\hline HCWs & 480 & 288(60.00) & 142(29.58) & $48(10.00)$ & $2(0.42)$ & & \\
\hline non-HCWs & 1020 & 748(73.33) & 201(19.71) & $55(5.39)$ & 16(1.57) & 36.03 & $<0.05$ \\
\hline \multicolumn{8}{|l|}{ Wuhan } \\
\hline HCWs & 134 & $65(48.51)$ & 45(33.58) & $24(17.91)$ & $\mathrm{O}(0.00)$ & & \\
\hline non-HCWs & 208 & $125(60.10)$ & $56(26.92)$ & $22(10.58)$ & $5(2.40)$ & 9.67 & $<0.05$ \\
\hline \multicolumn{8}{|c|}{ Other cities in Hubei except Wuhan } \\
\hline HCWs & 207 & $119(57.49)$ & $68(32.85)$ & 19(9.18) & $1(0.48)$ & & \\
\hline non-HCWs & 361 & 282(78.12) & $59(16.34)$ & $15(4.16)$ & $5(1.39)$ & 30.52 & $<0.05$ \\
\hline \multicolumn{8}{|c|}{ Other provinces in China except Hubei } \\
\hline HCWs & 139 & 104(74.82) & 29(20.86) & $5(3.60)$ & $1(0.72)$ & & \\
\hline non-HCWs & 451 & $341(75.61)$ & 86(19.07) & 18(3.99) & $6(1.33)$ & 0.56 & $>0.05$ \\
\hline
\end{tabular}

all residents were quarantined at home, the situation was chaotic and China was pressed the pause button. There was a widespread feeling of perceived life threat, extreme vulnerability, uncertainty and helplessness at that time. This fear spread within the public and also the hospitals, causing panic. As with any disaster, the influence and trauma of COVID-19 outbreak will lead to anxiety and depression, the overall levels of anxiety and depression obtained in this study were similar to the results of other studies $(23,24)$. Furthermore, we found that HCWs in Wuhan and other cities in Hubei except Wuhan had higher levels of anxiety and depression than non-HCWs, but there was no such difference in other provinces in China except Hubei. Compared with the other two regions, the HCWs in Wuhan was more anxious, and this anxiety might be caused by concerns about occupational exposure and wearing protective clothing for a long time daily. Compared with other provinces in China except Hubei, HCWs in the remaining two regions had more obvious depression, which might be associated with long days participating in epidemic 
TABLE 6 | Comparison of depression in different areas.

\begin{tabular}{|c|c|c|c|c|c|c|c|c|c|}
\hline & \multirow[b]{2}{*}{$N$} & \multicolumn{4}{|c|}{$\begin{array}{l}\text { Composition of HAMD } \\
\qquad n(\%)\end{array}$} & \multirow[t]{2}{*}{$x^{2}$} & \multirow[t]{2}{*}{$p^{*}$} & \multirow[t]{2}{*}{$x^{2}$} & \multirow[t]{2}{*}{ p\# } \\
\hline & & $<8$ & $8 \sim 20$ & $21 \sim 35$ & $>35$ & & & & \\
\hline \multicolumn{10}{|l|}{ HCWs } \\
\hline Wuhan & 134 & $65(48.51)$ & 45(33.58) & 24(17.91) & $\mathrm{O}(0.00)$ & & & & \\
\hline Other cities in Hubei except Wuhan & 207 & $119(57.49)$ & 68(32.85) & 19(9.18) & $1(0.48)$ & 6.79 & $>0.05$ & & \\
\hline Other provinces in China except Hubei & 139 & 104(74.82) & 29(20.86) & $5(3.60)$ & $1(0.72)$ & 25.82 & $<0.05^{\star}$ & 11.95 & $<0.05 \#$ \\
\hline \multicolumn{10}{|l|}{ non-HCWs } \\
\hline Wuhan & 208 & $125(60.10)$ & 56(26.92) & $22(10.58)$ & $5(2.40)$ & & & & \\
\hline Other cities in Hubei except Wuhan & 361 & 282(78.12) & 59(16.34) & $15(4.16)$ & $5(1.39)$ & 22.45 & $<0.05^{\star}$ & & \\
\hline Other provinces in China except Hubei & 451 & $341(75.61)$ & 86(19.07) & 18(3.99) & $6(1.33)$ & 20.07 & $<0.05^{\star}$ & 1.02 & $>0.05$ \\
\hline
\end{tabular}

$P^{\star}$ compared with Wuhan, P\# comparison between other cities in Hubei except Wuhan and other provinces in China except Hubei.

TABLE 7 | Risk of depression: logistic regression analysis.

\begin{tabular}{lcc}
\hline Variable & OR (95\% Cl) & $\boldsymbol{p}$ \\
\hline Male & $1.433(0.563-3.651)$ & 0.450 \\
Age & $0.996(0.957-1.036)$ & 0.826 \\
Whether you often worry about occupational & $1.587(0.816-3.086)$ & 0.173 \\
exposure (yes) & & \\
Whether you are worried about your family's & $0.350(0.079-1.543)$ & 0.165 \\
cross-infection because of you (no) & & \\
Daily wear protective clothing time (hours) & $1.100(1.040-1.163)$ & 0.001 \\
Participation in epidemic work(days) & $1.030(1.006-1.054)$ & 0.012 \\
\hline
\end{tabular}

$\mathrm{OR}$, odds ratio; $\mathrm{Cl}$, confidence interval.

work and wearing protective clothing for a long time daily. Meanwhile, $62.5 \%$ of HCWs were proud of their work. The anxiety and depression of non-HCWs in Wuhan were also the most serious.

We assessed both anxiety and depression levels in HCWs and non-HCWs. The results suggest that HCWs are worse off than non-HCWs in both anxiety and depression. This is not hard to predict, as the burden on HCWs is greater, they faced high risk of infection, insufficient contamination protection, overwork, negative emotions from patient, exhaustion, discrimination, isolation, etc. $(18,25,26)$. A literature review concluded that rescue workers have a higher incidence of post-traumatic stress disorder (PTSD) than the general population (27). In the early stages of the epidemic, the HCWs faced the uncertainty of encountering an undiagnosed COVID-19 patient every day, and there was no awareness of the need for systemic protection. When the epidemic broke out in full, the HCWs worked at the front line, and medical staff and resources were insufficient, their work intensity was high (28). As a result, both the occupational exposure and the risk of infection have greatly increased. Coupled with the fear of cross-infection among family members due to them, their psychological burden is heavier and pressure is greater. In addition, the differences in anxiety and depression levels between HCWs and non-HCWs in the three regions also implies that HCWs in Hubei are under more pressure and that the epidemic situation in Hubei is more serious which is consistent with the reality. The existing research has rarely investigated psychological status of HCWs and non-HCWs in Wuhan, other regions inside Hubei Province and regions outside Hubei Province during the COVID-19 outbreak $(5,12)$.

In order to clarify whether there is a difference in the psychological state of HCWs in different epidemic regions, we discussed the anxiety and depression of HCWs in Wuhan, other cities in Hubei except Wuhan and other provinces in China except Hubei. Our results suggest that during the outbreak of COVID-19 in mainland China, HCWs in high risk areas may have a higher prevalence of anxiety and depression. A study involving 34 hospitals showed that health care workers in Wuhan had more serious psychological problems (12). Other studies had also found that the proportion of frontline medical workers with anxiety and depression was significantly higher $(29,30)$. A recent comparative study showed that, at a time when confirmed COVID-19 cases rose sharply in Hong Kong and the epidemic in Hubei was under control, the prevalence of depression among health workers in Hong Kong (50.4\%) were more severe than in Hubei province (15.1\%) (25). It should be noted that the mental problems of both HCWs and general population in Hong Kong were reported to be exceptionally high (31). Since the performance of depressive symptoms is affected by comprehensive and complex internal and external factors, this discrepancy may due to the differences of regions, time points of the research, evaluation scales or sample sizes. Study on SARS have also shown that high-risk HCWs experience fatigue and poor sleep, worrying about health, accompanied by increased depression, anxiety, and post-traumatic stress scores (32). Moreover, we found that non-HCWs in Wuhan had a higher level of severe anxiety than other cities in Hubei except Wuhan, and a higher proportion of depression than the remaining two regions. In general, anxiety and depression levels among HCWs and non-HCWs in Wuhan, where the epidemic was most severe, were higher than elsewhere. Our conclusions were supported by a longitudinal study, which found that during SARS, anxiety levels were strongly correlated with the severity of the epidemic and closely mirrored the number of new cases per day $(4,25,33,34)$. 
Then, we assessed the perception of job among HCWs during the COVID-19 epidemic and the risk factors that caused different levels of anxiety and depression for HCWs in different regions were discussed. It was found that whether you often worry about occupational exposure and daily wear protective clothing time (hours) exerted significant independent effects for anxiety, and participation in epidemic work (days) and daily wear protective clothing time (hours) exerted significant independent effects for depression. Sex, age, and whether worried about family's crossinfection had no effect on the joint analysis. Fear of occupational exposure is a direct cause of anxiety. This not only increases their own risk of infection, but also threatens the health of their families. Wearing protective clothing for a long time daily reflects the high work intensity of HCWs, as well as an increased risk of infection, which not only causes anxiety but also induces depression. It is suggested that HCWs should not work too many hours a day $(18,26)$. The results also indicate that depression is directly related to long-term participation in epidemic work. In our survey, $86.57 \%$ of HCWs in Wuhan, $89.37 \%$ of HCWs in other cities in Hubei except Wuhan, and $71.22 \%$ of HCWs in other provinces in China except Hubei were worried about their family's cross-infection because of them. Given the high infectivity of the virus and its transmission through respiratory droplets and close contact, fear of inadvertently endangering members of family and loved ones was a widespread concern among HCWs (8). The HCWs were torn between their own responsibilities and this concern. Although the work of HCWs is high-risk, fortunately, we find that $62.5 \%$ of HCWs are proud of their work.

The study's discussion of causation is limited by its crosssectional nature. A further methodological limitation is the possible social expectation bias because of the use of self-report to assess psychiatric morbidity, some people may be inclined to underreport their psychopathology to avoid discrimination. We also cannot rule out the possibility that people with severe anxiety or depression was under-represented. Pre-COVID-19 anxiety and depression were not collected for HCWs and nonHCWs, so it was not possible to compare mental states before and after the outbreak. Finally, all residents in China are quarantined at home, so generalizations of our findings are limited, it may not apply to other countries. It would be meaningful to study the mental health status of HCWs and non-HCWs in different geographic regions at the beginning of the epidemic, the peak period, the remission period, and longer afterwards. Longitudinal studies with larger sample size are encouraged in future studies to conduct a more comprehensive assessment of this issue.

Despite these limitations, it is clear that HCWs, especially in Wuhan, have serious mental health problems. It is necessary to take relevant measures to treat or manage public anxiety and depression, for it may have a lasting effect after the epidemic ends (14). What's more, improving the psychological health of HCWs is of great significance for maintaining their physical health, keeping higher efficiency and working state, and controlling the epidemic $(35,36)$. The Chinese government has made some policies to address these psychological health problems and pay close attention to the psychological health of HCWs, such as caring for the elderly and children of HCWs to solve their worries, establishing a shift system so that front-line HCWs can take turns to rest, providing a professional psychological counseling platform, opening a public psychological hotline, and setting up psychological intervention teams to conduct psychological intervention and guidance to the masses through social platforms such as WeChat groups, etc.

In summary, when a new infectious disease breaks out, health care workers, especially where the epidemic is more severe, may bear heavier workloads and higher levels of anxiety and depression. Understanding the mental health response of COVID-19 may help prepare for future outbreaks of infectious diseases and improve the efficiency and quality of future crisis interventions.

\section{DATA AVAILABILITY STATEMENT}

The raw data supporting the conclusions of this article will be made available by the authors, without undue reservation.

\section{ETHICS STATEMENT}

The studies involving human participants were reviewed and approved by the Institutional Review Board (IRB) of Huazhong University of Science and Technology. Written informed consent from the participants was not required to participate in this study in accordance with the national legislation and the institutional requirements. The purpose and use of this research were explained at the beginning of the online questionnaire. All the participants provided online informed consent before entering the online questionnaire.

\section{AUTHOR CONTRIBUTIONS}

HC, GL, and FW designed the study. JL, LL, JZ, YH, CZ, and SH collected data. $\mathrm{CZ}$ and $\mathrm{YH}$ analyzed the data. FW and $\mathrm{YJ}$ administered the project. $\mathrm{HC}$ and $\mathrm{CZ}$ writing - original draft preparation. FW and GL writing - review \& editing. YJ and FW funding acquisition. All authors read and approved the final manuscript.

\section{FUNDING}

This study was funded by the National Natural Science Foundation of China $(81974218,81671064,81371222)$, the National Science \& Technology Fundamental Resources Investigation Project of China (2018FY100900) and the Innovative Scientific Research Foundation of HUST (540-5003540062, 540-5003540083).

\section{ACKNOWLEDGMENTS}

We thank to all the volunteers who participated in this study and thank to Zeqing Jiang for helping to sort out the data. 


\section{REFERENCES}

1. Yang Y, Li W, Zhang Q, Zhang L, Cheung T, Xiang Y-T. Mental health services for older adults in China during the COVID-19 outbreak. Lancet Psychiatry. (2020) 7:e19. doi: 10.1016/S2215-0366(20) 30079-1

2. Liu S, Yang L, Zhang C, Xiang Y-T, Liu Z, Hu S, et al. Online mental health services in China during the COVID-19 outbreak. Lancet Psychiatry. (2020) 7:e17-8. doi: 10.1016/s2215-0366(20)30077-8

3. Vindegaard $\mathrm{N}$, Benros ME. COVID-19 pandemic and mental health consequences: systematic review of the current evidence. Brain Behav Immun. (2020) 89:531-42. doi: 10.1016/j.bbi.2020. 05.048

4. Leung GM, Ho LM, Chan SK, Ho SY, Bacon-Shone J, Choy RY, et al. Longitudinal assessment of community psychobehavioral responses during and after the 2003 outbreak of severe acute respiratory syndrome in Hong Kong. Clin Infect Dis. (2005) 40:1713-20. doi: 10.1086/429923

5. Zhang WR, Wang K, Yin L, Zhao WF, Xue Q, Peng M, et al. Mental health and psychosocial problems of medical health workers during the COVID-19 epidemic in China. Psychother Psychosom. (2020) 89:242-50. doi: 10.1159/000507639

6. Bao Y, Sun Y, Meng S, Shi J, Lu L. 2019-nCoV epidemic: address mental health care to empower society. Lancet. (2020) 395:e37-8. doi: 10.1016/s0140-6736(20)30309-3

7. Su TP, Lien TC, Yang CY, Su YL, Wang JH, Tsai SL, et al. Prevalence of psychiatric morbidity and psychological adaptation of the nurses in a structured SARS caring unit during outbreak: a prospective and periodic assessment study in Taiwan. J Psychiatr Res. (2007) 41:119-30. doi: 10.1016/j.jpsychires.2005.12.006

8. An Y, Yang Y, Wang A, Li Y, Zhang Q, Cheung T, et al. Prevalence of depression and its impact on quality of life among frontline nurses in emergency departments during the COVID-19 outbreak. J Affect Disord. (2020) 276:312-5. doi: 10.1016/j.jad.2020.06.047

9. Qian G, Yang N, Ma AHY, Wang L, Li G, Chen X, et al. COVID-19 transmission within a family cluster by presymptomatic carriers in China. Clin Infect Dis. (2020) 71:861-2. doi: 10.1093/cid/ciaa316

10. Bai Y, Yao L, Wei T, Tian F, Jin DY, Chen L, et al. Presumed asymptomatic carrier transmission of COVID-19. JAMA. (2020) 323:1406-7. doi: 10.1001/jama.2020.2565

11. Maunder RG. Was SARS a mental health catastrophe? Gen Hosp Psychiatry. (2009) 31:316-7. doi: 10.1016/j.genhosppsych.2009. 04.004

12. Lai J, Ma S, Wang Y, Cai Z, Hu J, Wei N, et al. Factors Associated with mental health outcomes among health care workers exposed to coronavirus disease 2019. JAMA Netw Open. (2020) 3:e203976. doi: 10.1001/jamanetworkopen.2020.3976

13. Pappa S, Ntella V, Giannakas T, Giannakoulis VG, Papoutsi E, Katsaounou P. Prevalence of depression, anxiety, and insomnia among healthcare workers during the COVID-19 pandemic: a systematic review and metaanalysis. Brain Behav Immun. (2020) 88:901-7. doi: 10.1016/j.bbi.2020. 05.026

14. Liu X, Kakade M, Fuller CJ, Fan B, Fang Y, Kong J, et al. Depression after exposure to stressful events: lessons learned from the severe acute respiratory syndrome epidemic. Compr Psychiatry. (2012) 53:15-23. doi: 10.1016/j.comppsych.2011.02.003

15. Liu X, Luo WT, Li Y, Li CN, Hong ZS, Chen HL, et al. Psychological status and behavior changes of the public during the COVID-19 epidemic in China. Infect Dis Poverty. (2020) 9:58. doi: 10.1186/s40249-020-00678-3

16. Yu BY, Yeung WF, Lam JC, Yuen SC, Lam SC, Chung VC, et al. Prevalence of sleep disturbances during COVID-19 outbreak in an urban Chinese population: a cross-sectional study. Sleep Med. (2020) 74:18-24. doi: 10.1016/j.sleep.2020.07.009

17. Wang C, Pan R, Wan X, Tan Y, Xu L, Ho CS, et al. Immediate psychological responses and associated factors during the initial stage of the 2019 coronavirus disease (COVID-19) epidemic among the general population in China. Int J Environ Res Public Health. (2020) 17:1729. doi: 10.3390/ijerph17051729
18. Kang L, Li Y, Hu S, Chen M, Yang C, Yang BX, et al. The mental health of medical workers in Wuhan, China dealing with the 2019 novel coronavirus. Lancet Psychiatry. (2020) 7:e14. doi: 10.1016/S2215-0366(20) 30047-X

19. Zimmerman M, Thompson JS, Diehl JM, Balling C, Kiefer R. Is the DSM-5 Anxious Distress Specifier Interview a valid measure of anxiety in patients with generalized anxiety disorder: a comparison to the Hamilton Anxiety Scale. Psychiatry Res. (2020) 286:112859. doi: 10.1016/j.psychres.2020.112859

20. Shear MK, Bilt JV, Rucci P, Endicott J, Lydiard B, Otto MW, et al. Reliability and validity of a structured interview guide for the hamilton anxiety rating scale (scale-A). Depress Anxiety. (2001) 13:166-78.

21. Laenen A, Alonso A, Molenberghs G, Vangeneugden T, Mallinckrodt $\mathrm{CH}$. Using longitudinal data from a clinical trial in depression to assess the reliability of its outcome scales. J Psychiatr Res. (2009) 43:730-8. doi: 10.1016/j.jpsychires.2008.09.010

22. Eurosurveillance Editorial Team. Note from the editors: World Health Organization declares novel coronavirus (2019-nCoV) sixth public health emergency of international concern. Euro Surveill. (2020) 25:200131e. doi: 10.2807/1560-7917.ES.2020.25.5.200131e

23. Rajkumar RP. COVID-19 and mental health: a review of the existing literature. Asian J Psychiatr. (2020) 52:102066. doi: 10.1016/j.ajp.2020.1 02066

24. Salari N, Hosseinian-Far A, Jalali R, Vaisi-Raygani A, Rasoulpoor S, Mohammadi $\mathrm{M}$, et al. Prevalence of stress, anxiety, depression among the general population during the COVID-19 pandemic: a systematic review and meta-analysis. Global Health. (2020) 16:57. doi: 10.1186/s12992-020-0 0589-w

25. Lam SC, Arora T, Grey I, Suen LKP, Huang EY, Li D, et al. Perceived risk and protection from infection and depressive symptoms among healthcare workers in Mainland China and Hong Kong during COVID-19. Front Psychiatry. (2020) 11:686. doi: 10.3389/fpsyt.2020. 00686

26. Theorell T. COVID-19 and working conditions in health care. Psychother Psychosom. (2020) 89:193-4. doi: 10.1159/000 507765

27. Neria Y, Nandi A, Galea S. Post-traumatic stress disorder following disasters: a systematic review. Psychol Med. (2008) 38:467-80. doi: 10.1017/S0033291707001353

28. Ran L, Chen X, Wang Y, Wu W, Zhang L, Tan X. Risk factors of healthcare workers with corona virus disease 2019: a retrospective cohort study in a designated hospital of Wuhan in China. Clin Infect Dis. (2020) ciaa287. doi: 10.1093/cid/ciaa287. [Epub ahead of print].

29. Chen J, Liu X, Wang D, Jin Y, He M, Ma Y, et al. Risk factors for depression and anxiety in healthcare workers deployed during the COVID19 outbreak in China. Soc Psychiatry Psychiatr Epidemiol. (2020) 1-9. doi: 10.1007/s00127-020-01954-1. [Epub ahead of print].

30. Wang H, Huang D, Huang H, Zhang J, Guo L, Liu Y, et al. The psychological impact of COVID-19 pandemic on medical staff in Guangdong, China: a cross-sectional study. Psychol Med. (2020) 1-9. doi: 10.1017/S0033291720002561

31. Bressington D, Cheung TCC, Lam SC, Suen LKP, Fong TKH, Ho HSW, et al. Association between depression, health beliefs and face mask use during the COVID-19 pandemic. Front Psychiatry. (2020) 11:1075. doi: $10.3389 /$ fpsyt.2020.571179

32. Lu YC, Shu BC, Chang YY, Lung FW. The mental health of hospital workers dealing with severe acute respiratory syndrome. Psychother Psychosom. (2006) 75:370-5. doi: 10.1159/000095443

33. Xiaoming $\mathrm{X}$, Ming $\mathrm{A}$, Su H, Wo W, Jianmei C, Qi Z, et al. The psychological status of 8817 hospital workers during COVID-19 Epidemic: a cross-sectional study in Chongqing. J Affect Disord. (2020) 276:555-61. doi: 10.1016/j.jad.2020.07.092

34. Liu Y, Chen H, Zhang N, Wang X, Fan Q, Zhang Y, et al. Anxiety and depression symptoms of medical staff under COVID-19 epidemic in China. J Affect Disord. (2020) 278:144-8. doi: 10.1016/j.jad.2020.09.004

35. Schwartz J, King CC, Yen MY. Protecting Health Care Workers during the COVID-19 Coronavirus Outbreak -Lessons from Taiwan's SARS response. Clin Infect Dis. (2020) 71:858-860. doi: 10.1093/cid/ciaa255 
36. Chen Q, Liang M, Li Y, Guo Y, Fei D, Wang L, et al. Mental health care for medical staff in China during the COVID-19 outbreak. Lancet Psychiatry. (2020) 7:e15-6. doi: 10.1016/S2215-0366(20)30078-X

Conflict of Interest: The authors declare that the research was conducted in the absence of any commercial or financial relationships that could be construed as a potential conflict of interest.
Copyright $\odot 2020$ Cao, Zuo, Li, Huang, Li, Huang, Zhao, Liu, Jiang and Wang. This is an open-access article distributed under the terms of the Creative Commons Attribution License (CC BY). The use, distribution or reproduction in other forums is permitted, provided the original author $(s)$ and the copyright owner(s) are credited and that the original publication in this journal is cited, in accordance with accepted academic practice. No use, distribution or reproduction is permitted which does not comply with these terms. 